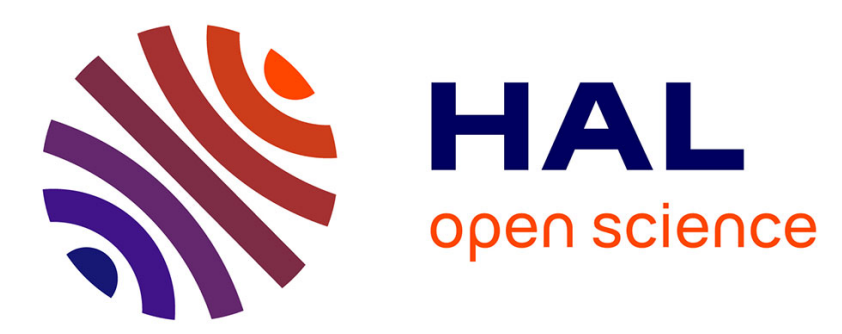

\title{
Distributed Downlink Resource Allocation in Cellular Networks through Spatial Adaptive Play
}

\author{
Chandramani Singh, Chung Shue Chen
}

\section{To cite this version:}

Chandramani Singh, Chung Shue Chen. Distributed Downlink Resource Allocation in Cellular Networks through Spatial Adaptive Play. ITC25: the 25th International Teletraffic Congress, Sep 2013, Shanghai, China. hal-00842077

\section{HAL Id: hal-00842077 https://hal.inria.fr/hal-00842077}

Submitted on 7 Jul 2013

HAL is a multi-disciplinary open access archive for the deposit and dissemination of scientific research documents, whether they are published or not. The documents may come from teaching and research institutions in France or abroad, or from public or private research centers.
L'archive ouverte pluridisciplinaire HAL, est destinée au dépôt et à la diffusion de documents scientifiques de niveau recherche, publiés ou non, émanant des établissements d'enseignement et de recherche français ou étrangers, des laboratoires publics ou privés. 


\section{Distributed Downlink Resource Allocation in Cellular Networks through Spatial Adaptive Play}

\author{
Chandramani K. Singh \\ TREC, INRIA-ENS, Paris, France \\ INRIA-Alcatel-Lucent Bell Labs Joint Research Center \\ Email: chandramani.singh@inria.fr
}

\author{
Chung Shue Chen \\ Alcatel-Lucent Bell Labs \\ Centre de Villarceaux, 91620 Nozay, France \\ Email: cs.chen@alcatel-lucent.com
}

\begin{abstract}
In this work, we develop mathematical and algorithmic tools for distributed resource allocation in downlink of mobile cellular networks. Our algorithms perform power allocation, subcarrier selection and base station association simultaneously. We aim to maximize the aggregate utility of all the users where users' utilities can be arbitrary increasing functions of their throughputs; this allows us to capture both elastic and inelastic traffics. Our solution is via framing the problem as a potential game among users. We propose a highly scalable, asynchronous algorithm that provably converges to a Nash equilibrium of this game. This algorithm requires only local measurements, limited communication between neighboring nodes and limited computation. This algorithm may at times stuck at a local maximum. To alleviate this problem, we propose an enhanced randomized algorithm based on spatial adaptive play that provably converges to a system optimal resource allocation. We also present simulation results to illustrate convergence and performance of the proposed algorithms.
\end{abstract}

\section{INTRODUCTION}

Wireless networks have experienced tremendous growth in terms of size, scope, and traffic demand, and this growth continues unabated worldwide. Efficient management of resources is essential for the success of wireless cellular systems. Specially, autonomic management is desirable to relax the heavy requirement of human efforts in conventional network planning and optimization tasks [1]. In 4G and future cellular mobile radio systems, network self-organization and selfoptimization are among the key targets [2].

Optimal power control, BS association and channel allocation are central to the efficient operation of cellular networks. For example, power control not only helps maintaining quality of service (QoS) but also enhances users' battery lives (in uplink). In addition, it also reduces the network interference, thus maximizing spatial spectrum reuse. Similarly, clever BS association and channel allocation facilitate load balancing in the wake of user mobility and traffic variability. Efficient resource allocation becomes even more crucial with proliferation of heterogeneous networks (comprising of femtocells and picocells along with the traditional macrocells) and with provision of finer resource granularity in future OFDMA based networks (e.g., resource blocks in LTE systems) that facilitates packing efficiency and exploitation of time/frequency channel selectivity. Nonetheless, such flexibility also allows meeting diverse class of QoS requirements ranging from initial voicecentric service to recent high data rate broadband services.

System-wide radio resource optimization is however usually quite challenging. Today's networks typically involve large numbers of users and cells (and also other resource units). Users' utilities can often be nonconcave (e.g., in case of voice/video traffic), and there may be integrality constraints associated with BS/carrier assignments. Even in case of elastic traffic, transmission power optimization over multiple interfering links becomes a non-convex problem (see e.g., [3] [4]). Finally, thanks to channel randomness and users' mobility, resource allocation needs to be performed at a fast time scale, asynchronously and using only local information. In view of these complicacies, the resource allocation problem at hand is not amenable to standard optimization techniques. We take a slightly nontraditional route in which we model the problem using the theory of non-cooperative games, and propose a distributed algorithm based on spatial adaptive play (SAP) which is intrinsically related to Gibbs sampling in statistical mechanics, as highlighted in Section II-B.

There is a vast literature on distributed power control and BS association in wireless cellular networks. Initial attempts by Foschini and Miljanic [5], Yates [6] and Hanly [7] were focused on uplink of single carrier networks; Farrokhi et al. [8] addressed the downlink counterpart. Baccelli et al. [9] studied power control to achieve optimal delay-power tradeoff in the context of elastic traffic. Bu et al. [10] showed NP-hardness of generalized proportional fair scheduling in $3 \mathrm{G}$ wireless data networks. Huang et al. [11] considered scheduling and resource allocation for the downlink of OFDM-based wireless networks. Wu et al. [12] introduced a fractional power reuse scheme to multi-cell cellular networks, which was shown to have bigger capacity region as compared to the traditional frequency reuse schemes. Using a similar idea, Stolyar and Viswanathan [13] proposed an algorithm for subcarrier and power allocation in downlink of OFDMA systems. Hou and Chen [14] studied weighted proportional fair resource allocation for the downlink of LTE networks. In particular, they proposed algorithms that jointly perform scheduling, power control and client association.

Non-cooperative games are natural tools for analysis and design of distributed resource allocation algorithms. Scutari et al. [15] and Heikkinen [16] modeled distributed power control problems in CDMA networks as potential games, while Altman and Altman [17] showed that many of the cellular power control algorithms can be modeled as submodular games. Alpacan et al. [18] and Saraydar et al. [19] framed power control as non-cooperative games, and used pricing to attain efficient power allocations. Singh et al. [20] used the 
framework of potential games to address joint power control and BS association for the uplink of multichannel, multicell cellular networks; BS association in downlink was studied by Jiang et al. [21].

Gibbs sampling based self organization techniques for wireless networks have only recently generated interest. Kauffmann et al. [22] proposed distributed channel selection and node association algorithms in the context of multi-channel 802.11 WLANs. Chen and Baccelli [23] derived Gibbs sampling based power control and BS association algorithms for reuse-1 cellular networks; their algorithms are aimed at providing delay fairness. Borst et al. [24] also developed a joint power control, BS association and scheduling algorithm in OFDMA cellular networks. Our work can be seen as extension of [23] to general utility functions. On the other hand, our proposed algorithm is similar in flavor to the one developed in [24]. We delineate our work from [24] in the following section.

\section{A. Our Contribution}

We study distributed resource allocation in the downlink of multichannel, multicell cellular networks. We propose an algorithm that optimizes the aggregate utility of all the users, where the users can have distinct and arbitrary individual utilities. The following is a preview of our main contributions.

1) We develop a learning based algorithm (motivated by "spatial adaptive play") for joint downlink power control, BS assignment and channel allocation. Our algorithm drives the network into the optimal configuration, and the framework accommodates arbitrary utility functions. Though our algorithm is similar in flavor to the one proposed by Borst et al. [24], we follow a different approach to arrive at this. Borst et al. start with a graph representation of the network, identify cliques and associated potential functions and local energy functions. They further analyze the underlying random field and invoke the Gibbs-Markov equivalence before claiming the convergence of their constrained Gibbs sampler (CGS) algorithm. On the other hand, we exploit a direct connection between potential games and spatial adaptive play. We believe that our approach is more straightforward, and it lets us focus on the distributed implementation aspect.

2) As a byproduct of the game-theoretic approach, we also get deterministic algorithms (best response based) that provably converge, albeit to a local optimum. But these algorithms exhibit much faster convergence rate and attain performance close to the global optimal (see the numerical results in Section VI). In practice, the resource allocation has to be achieved at a fast time scale which makes these deterministic algorithms extremely useful.

3) We develop distributed protocols in which BSs need to exchange information only with their neighbors. This is noticeable improvement over the protocols of Borst et al. [24] who require BSs to communicate with two-hop neighbors as well.
The rest of the paper is organized as follows. In section II, we briefly discuss the concepts of potential games and spatial adaptive play. Here we also highlight the analogy between the latter and Gibbs sampling. In Section III, we describe the system model and the problem formulation. We develop an optimal resource allocation algorithm in Section IV. In Section V, we present a distributed protocol, and we also discuss the information exchange requirements and complexity of the proposed protocol. Section VI contains numerical studies. Finally, we conclude the paper in Section VII where we indicate a few future directions.

\section{Potential Games and Learning}

\section{A. Potential Games}

A non-cooperative strategic form game $\left(\mathcal{N},\left(\mathcal{S}_{i}, i \in \mathcal{N}\right)\right.$, $\left.\left(v_{i}, i \in \mathcal{N}\right)\right)$ consists of a set of players $\mathcal{N}=\{1, \ldots, N\}$. Each player $i$ is accompanied by a strategy set $\mathcal{S}_{i}$ and a payoff function $v_{i}: \times_{i=1}^{N} \mathcal{S}_{i} \rightarrow \mathbb{R}$. In this work, we assume all strategy sets to be finite. A strategy profile $\mathbf{s}=\left(s_{i}, i=1, \ldots, N\right)$ prescribes a strategy $s_{i}$ for every player $i \in \mathcal{N}$. For $\mathbf{s}=$ $\left(s_{i}, i=1, \ldots, N\right)$, denote $\mathbf{s}_{-i}:=\left(s_{1}, \ldots, s_{i-1}, s_{i+1}, \ldots, s_{N}\right)$ and $\left(s_{i}^{\prime}, \mathbf{s}_{-i}\right):=\left(s_{1}, \ldots, s_{i-1}, s_{i}^{\prime}, s_{i+1}, \ldots, s_{N}\right)$.

Definition 2.1: Nash Equilibrium: For a strategy profile $\mathbf{s}$, a player $i$ 's best response, $\mathcal{R}_{i}(\mathbf{s}) \subseteq \mathcal{S}_{i}$, is defined as $\mathcal{R}_{i}(\mathbf{s}):=$ $\operatorname{argmax}_{s_{i}^{\prime} \in \mathcal{S}_{i}} v_{i}\left(s_{i}^{\prime}, \mathbf{s}_{-i}\right)$. A strategy profile $\mathbf{s}$ is said to be a Nash Equilibrium for the game if $s_{i} \in \mathcal{R}_{i}(\mathbf{s})$ for all $i \in \mathcal{N}$.

Definition 2.2: Exact Potential Game: A game $\left(\mathcal{N},\left(\mathcal{S}_{i}, i \in\right.\right.$ $\left.\mathcal{N}),\left(v_{i}, i \in \mathcal{N}\right)\right)$ is said to be an exact potential game if there exists a function $V: \times_{i \in \mathcal{N}} \mathcal{S}_{i} \rightarrow \mathbb{R}$, known as a potential function, that satisfies

$$
v_{i}\left(s_{i}^{\prime}, \mathbf{s}_{-i}\right)-v_{i}(\mathbf{s})=V\left(s_{i}^{\prime}, \mathbf{s}_{-i}\right)-V(\mathbf{s})
$$

for all $i \in \mathcal{N}, s_{i}^{\prime} \in \mathcal{S}_{i}, \mathbf{s} \in \times_{i \in \mathcal{N}} \mathcal{S}_{i}$.

In this work, we refer to exact potential games simply as potential games. Clearly, all maximizers of a potential function $V$ are Nash equilibria of the potential game. Thus, all potential games $\left(\mathcal{N},\left(\mathcal{S}_{i}, i \in \mathcal{N}\right),\left(v_{i}, i \in \mathcal{N}\right)\right)$ admit at least one Nash equilibrium. On account of their finiteness, they also have the finite improvement path (FIP) property, i.e., they do not contain improvement cycles (see Monderer and Shapley [25, Lemma 2.3]). Thus, in a finite potential game when players update as per the better response strategy or best response strategy, round-robin, random or asynchronous update processes converge to a Nash equilibrium (see Neel [26, Chapter 5] for convergence under asynchronous update process). To illustrate strategy updates, let us consider a slotted system and asynchronous best response update process. For epochs $t=0,1,2, \ldots$, let $\mathbf{s}(t)$ be the strategy profile at $t$. At $t+1$, each player $i$ updates its strategy with probability $\epsilon_{i} \in(0,1)$; thus for any subset of players there is a strictly positive probability of these players updating their strategies simultaneously. The player $i$ chooses its strategy from the set $\operatorname{argmax}_{s_{i}^{\prime} \in \mathcal{S}_{i}} v_{i}\left(s_{i}^{\prime}, \mathbf{s}_{-i}(t)\right)$. All other players repeat their strategies, i.e., $\mathbf{s}_{-i}(t+1)=\mathbf{s}_{-i}(t)$.

Often the potential functions are social performance measures. In such a case, any desirable operating point should be 
a maximizer of the potential function. While all maximizers of the potential function are Nash equilibria, the greedy (better response or best response) updates may lead to an equilibrium that does not maximize the potential function. We then resort to less greedy, explorative learning algorithms. We describe one such algorithm in Section II-B.

\section{B. A Learning Algorithm}

We now describe a learning algorithm known as spatial adaptive play (SAP), which was proposed by Young [27]. The basic idea in this algorithm is to keep a possibility to escape after encountering a local maximizer (strategy profile) of the potential function. The escape probabilities are determined by an exploration parameter which is controlled such that the updates settle at a global maximizer.

We can again consider round-robin, random or asynchronous update processes. In the following we describe the random update version. As before, let $\mathbf{s}(t)$ be the strategy profile at $t$. At $t+1$, a player $i$ is randomly chosen to update its strategy; all users have strictly positive probabilities of being chosen. The player $i$ randomly selects a strategy from $\mathcal{S}_{i}$ according to the following probability distribution $\pi_{i}^{t+1}$ which is a function of $\mathbf{s}_{-i}(t)$ : for all $s_{i} \in \mathcal{S}_{i}, s_{i}(t+1)=s_{i}$ with probability

$$
\pi_{i}^{t+1}\left(s_{i}, \mathbf{s}_{-i}(t)\right)=\frac{e^{\beta v_{i}\left(s_{i}, \mathbf{s}_{-i}(t)\right)}}{\sum_{s_{i}^{\prime} \in \mathcal{S}_{i}} e^{\beta v_{i}\left(s_{i}^{\prime}, \mathbf{s}_{-i}(t)\right)}}
$$

for some exploration parameter $\beta \geq 0$. The constant $\beta$ determines how likely player $i$ is to select a sub-optimal strategy. If $\beta=0$, player $i$ selects any strategy $s_{i} \in \mathcal{S}_{i}$ with equal probability. For high values of $\beta$, player $i$ will select a best response with high probability. All other players repeat their strategies, i.e., $\mathbf{s}_{-i}(t+1)=\mathbf{s}_{-i}(t)$. The spatial adaptive play converges geometrically fast to the following stationary distribution on strategy profiles [27, Theorem 6.2]:

$$
\pi(\mathbf{s})=\frac{e^{\beta V(\mathbf{s})}}{\sum_{\mathbf{s}^{\prime} \in \mathcal{S}} e^{\beta V\left(\mathbf{s}^{\prime}\right)}}
$$

for all $\mathbf{s} \in \mathcal{S} .^{1}$ Notice that, for positive $\beta$, this distribution puts more mass on strategy profiles that lead to higher values of potential function. Also, small values of $\beta$ lead to slower convergence. In practice, we start with a small $\beta$ and increase it in an appropriate way (e.g., as $\beta(t)=\log (1+t)$ ) as the update process proceeds. If $\beta(t) \rightarrow \infty$, the resulting steady state distribution $\pi$ puts entire mass at the strategy profiles that maximize the potential function ( $\pi$ is dirac distribution if there is only one maximizer). Thus, starting from any arbitrary state, the updates drive the network to an optimal strategy profile.

Remark 2.1: In statistical mechanics literature, the above local transition based learning (with fixed $\beta$ ) is referred to as Gibbs sampling. The negative of the potential function $V(\cdot)$ is called global energy of the Gibbs sampler, and the steady state distribution in (2) is called the Gibbs distribution associated with $V(\cdot)$. The denominator of (2) is called partition function,

\footnotetext{
${ }^{1}$ For sufficiently large $t, \pi(\mathbf{s})$ approximates the probability that $\mathbf{s}(t)=\mathbf{s}$.
}

the inverse of the exploration parameter $\beta$ is called temperature, and gradual increase of $\beta$ with time is called cooling. The update algorithm with increasing $\beta$ is called simulated annealing.

Remark 2.2: This approach exploits a generic connection between best response strategies (in potential games) and Gibbs sampling. In wireless network literature a plurality of problems have been approached using the potential game framework. Evidently, Gibbs sampler based randomized algorithms are applicable in all such cases and come to rescue if the systems can stuck at local minima.

\section{System Model AND PROBLEM Formulation}

\section{A. System Model}

We now describe the model adopted in this work. We consider a cellular network consisting of several BSs and users. All the cells employ same set of channels for communication. ${ }^{2}$ However, uplink and downlink communications take place over disjoint sets of channels, and so, there is no interference between the uplink and downlink. We consider only the downlink in the present paper.

Let $\mathcal{B}$ and $\mathcal{N}$ denote the set of BSs and the set of users, respectively, and let $\mathcal{C}$ denote the common set of channels used for downlink communication by all the BSs. The BSs' transmit powers for the connected users are chosen from a discrete set $\mathcal{P}$. Let $l(i, b, c)$ denote the power attenuation from $\mathrm{BS} b$ to user $i$ on channel $c$. Let $\alpha\left(c, c^{\prime}\right)$ denote the orthogonality factor that determines the effect of transmission on channel $c^{\prime}$ on the received signal at a user that is receiving on channel $c$. Clearly, $\alpha\left(c, c^{\prime}\right) \leq 1$ for all $c, c^{\prime} \in \mathcal{C}$. For example, $\alpha\left(c, c^{\prime}\right)=0$ if $c$ and $c^{\prime}$ are completely orthogonal, and $\alpha(c, c)=1$ for all $c \in \mathcal{C}$. We can also assume that

$$
\alpha\left(c, c^{\prime}\right)=\alpha\left(c^{\prime}, c\right) .
$$

Let $w_{i}\left(c_{i}\right)$ denote user $i$ 's receiver noise power on channel $c_{i}$.

The BSs periodically broadcast pilot signals of a priory fixed power. Let $\mathcal{B}_{i} \subseteq \mathcal{B}$ be the set of BSs such that the received pilot signal power at user $i$ from a BS in $\mathcal{B}_{i}$ is greater than a certain threshold, say $\theta$. Typically, user $i$ connects to a BS in $\mathcal{B}_{i}$. We refer to the users $\left\{i: b \in \mathcal{B}_{i}\right\}$ as potential users of BS $b$. We view the resource allocation as BSs jointly determining user-BS associations and then assigning channels and powers to users, where a BS can assign its resources only to its potential users. ${ }^{3}$

Let $b_{i}$ be the BS to which user $i$ connects, and let $c_{i}$ and $p_{i}$ be the channel and power used by $b_{i}$ respectively to transmit data to $i$. We use notation $s_{i}:=\left(b_{i}, c_{i}, p_{i}\right)$, and we refer to $\mathbf{s}=\left(s_{i}, i \in \mathcal{N}\right)$ as a resource allocation profile. Under a resource allocation profile $\mathbf{s}$, the signal to interference plus noise ratio (SINR) at user $i$ is

\footnotetext{
${ }^{2} \mathrm{~A}$ cell refers to a BS and the associated users.

${ }^{3}$ We propose a generic framework to address a fundamental resource allocation problem in cellular networks. Here, unlike 3GPP-LTE OFDMA systems, we allow more than one user to associate with a BS over the same subcarrier. We also consider only maximum power constraint per subcarrier per user rather than modeling total power constraints of BSs. However, our framework can be extended to incorporate these constraints
} 


$$
\operatorname{SINR}_{i}(\mathbf{s})=\frac{l\left(i, b_{i}, c_{i}\right) p_{i}}{\sum_{j \neq i} \alpha\left(c_{i}, c_{j}\right) l\left(i, b_{j}, c_{j}\right) p_{j}+w_{i}\left(c_{i}\right)} .
$$

Under the additive white Gaussian noise (AWGN) model, the rate (in bits/s/Hz) obtained by user $i$ can be expressed as

$$
r_{i}(\mathbf{s})=K \log \left(1+\operatorname{sinR}_{i}(\mathbf{s})\right),
$$

where $K$ is a constant that depends on the width of the downlink channel.

In the following discussion we do not show the dependence of SINRs (or, rates) on the underlying resource allocation profile if this omission does not cause ambiguity.

\section{B. Utility Functions}

We consider utility functions that are arbitrary but real, continuous and increasing functions of users' rates. Different users can have different utility functions. Observe that users' rates are continuously increasing functions of their SINRs. Thus users utilities can be expressed directly as functions of their SINRs. Let $U_{i}: \mathbb{R}_{+} \rightarrow \mathbb{R}$ denote the function mapping user $i$ 's SINR to its utility. Then we are interested in maximizing the following aggregate utility

$$
U(\mathbf{s})=\sum_{i \in \mathcal{N}} U_{i}\left(\operatorname{SINR}_{i}\right) .
$$

Notice that users' SINRs depend on BS associations, channel assignments and powers used by BSs for transmission. The BS associations, channel assignments and powers used constitute the decision variables in the above optimization problem.

We now give a few examples that might be of particular interest.

1) Aggregate throughput maximization: In this case, $U_{i}\left(\operatorname{SINR}_{i}\right)=r_{i}\left(\operatorname{SINR}_{i}\right)$ for all $i \in \mathcal{N}$.

2) Proportional fair resource allocation: Here,

$$
U_{i}\left(\operatorname{SINR}_{i}\right)=\log \left(\log \left(1+\operatorname{SINR}_{i}\right)\right)
$$

for all $i \in \mathcal{N}$.

3) Aggregate Delay minimization: This solution for resource allocation is intermediate between proportional and max-min fairness. It minimizes the sum of the inverse of throughput, i.e.,

$$
U_{i}\left(\operatorname{SINR}_{i}\right)=-\frac{1}{\log \left(1+\operatorname{SINR}_{i}\right)}
$$

for all $i \in \mathcal{N}$. Chen and Baccelli [23] have studied this problem under the low SINR regime, i.e., under the assumption that $\log \left(1+\operatorname{SINR}_{i}\right) \approx \operatorname{SINR}_{i}$. Thus, they minimize $\sum_{i \in \mathcal{N}} \frac{1}{\operatorname{SINR}_{i}}$.

\section{An SINR Approximation}

Observe that a user's received SINR depends on the joint resource allocation across the entire network. Thus optimal resource allocation would require information exchange between all the BSs and users. In order to arrive at a distributed algorithm, we now approximate the interference at user $i$ by only accounting for transmissions of BSs in $\mathcal{B}_{i}$; recall that the received pilot signal powers at user $i$ from all other
BSs are less than $\theta$. Thus, these BSs' transmissions can be neglected. More formally, we introduce notation $I_{i}(\mathbf{s})$ for the approximate interference at user $i$ :

$$
I_{i}(\mathbf{s}):=\sum_{\substack{j \neq i \\ b_{j} \in \mathcal{B}_{i}}} \alpha\left(c_{i}, c_{j}\right) l\left(i, b_{j}, c_{j}\right) p_{j} .
$$

We now express the received SINR as

$$
\operatorname{SINR}_{i}(\mathbf{s})=\frac{l\left(i, b_{i}, c_{i}\right) p_{i}}{I_{i}(\mathbf{s})+w_{i}\left(c_{i}\right)} .
$$

We use this approximate SINR expression in the following analysis. Notice that $i$ 's approximate $\operatorname{SINR}, \operatorname{SINR}_{i}(\mathbf{s})$, and thus $U_{i}\left(\operatorname{SINR}_{i}\right)$ depend on $\mathbf{s}$ only through $\left(s_{j}, j: b_{j} \in \mathcal{B}_{i}\right)$. We use this observation in our non-cooperative game formulation in the next section.

\section{Optimal Resource Allocation}

\section{A. A Neighborhood Structure}

We first introduce a neighborhood structure that facilitates development of a decentralized resource allocation algorithm as described in Section V.

A user $j$ is called neighbor of user $i$ if there exists a BS $b \in \mathcal{B}_{j}$ such that $b$ 's pilot signal is received by $i$ with received power exceeding $\theta$, i.e., $b \in \mathcal{B}_{i}$. Let $\mathcal{N}_{i}$ be the set of all neighbors of $i$. Formally,

$$
\mathcal{N}_{i}=\left\{j: \mathcal{B}_{i} \cap \mathcal{B}_{j} \neq \emptyset\right\} .
$$

By definition, $\mathcal{N}_{i}$ includes $i$. In our development we use the following two properties of sets $\mathcal{N}_{i}$ :

1) For any pair $(i, j), j \in \mathcal{N}_{i}$ if and only if $i \in \mathcal{N}_{j}$.

2) Given $\left(s_{j}: j \in \mathcal{N}_{i}\right), U_{i}(\mathbf{s})$ is independent of other components of $\mathbf{s}$.

Let us consider two BSs $b$ and $b^{\prime}$. We call $b^{\prime}$ a neighbor of $b$ if there exist users $i$ and $j$ such that $b \in \mathcal{B}_{i}, b^{\prime} \in \mathcal{B}_{j}$, and $j \in \mathcal{N}_{i}$. Notice that a user $i$ 's resource allocation update, say from $s_{i}$ to $s_{i}^{\prime}$, affects its own utility and the utilities of users $j$ for which either $b_{i} \in \mathcal{B}_{j}$ or $b_{i}^{\prime} \in \mathcal{B}_{j}$ or both. Clearly, a BS needs to exchange information only with its neighbors in order to make its resource allocation decisions. We will elaborate on the message exchanges in Section V-A.

\section{B. Potential Game Formulation}

We frame the joint resource allocation problem as the following non-cooperative game.

Players: The users are the players, i.e., $\mathcal{N}$ is the set of players.

Strategies: For user $i$, a strategy is a tuple $s_{i}=\left(b_{i}, c_{i}, p_{i}\right)$, and its strategy set is $\mathcal{S}_{i}:=\mathcal{B}_{i} \times \mathcal{C} \times \mathcal{P}$. A joint strategy $\mathbf{s}=$ $\left(s_{i}, i \in \mathcal{N}\right)$ belongs to the joint strategy space $\mathcal{S}:=\times_{i \in \mathcal{N}} \mathcal{S}_{i}$.

Payoffs: User $i$ 's payoff function $V_{i}: \mathcal{S} \rightarrow \mathbb{R}$ is defined as

$$
V_{i}(\mathbf{s}):=\sum_{j \in \mathcal{N}_{i}} U_{j}(\mathbf{s}) .
$$

In the following, we refer to the above game as the strategic form game $\left(\mathcal{N},\left(\mathcal{S}_{i}, i \in \mathcal{N}\right),\left(V_{i}, i \in \mathcal{N}\right)\right)$. 
Remark 4.1: While depicting users as players, we do not suggest that the users make resource allocation decisions. Our game formulation is simply a mechanism to develop a distributed resource allocation algorithm as explained in Section V.

Theorem 4.1: The finite strategic form game $\left\{\mathcal{N},\left(\mathcal{S}_{i}, i \in\right.\right.$ $\left.\mathcal{N}),\left(V_{i}, i \in \mathcal{N}\right)\right)$ is a potential game and thus admits the FIP.

Proof: Let players use a strategy $\mathbf{s}=\left(s_{i}, i \in \mathcal{N}\right)$. Consider a player $i$, and assume that it changes its strategy from $s_{i}$ to $s_{i}^{\prime}$. Now

$$
\begin{aligned}
& U\left(s_{i}^{\prime}, \mathbf{s}_{-i}\right)-U(\mathbf{s}) \\
& =\sum_{j \in \mathcal{N}}\left(U_{j}\left(s_{i}^{\prime}, \mathbf{s}_{-i}\right)-U_{j}(\mathbf{s})\right) \\
& \stackrel{(*)}{=} \sum_{j \in \mathcal{N}_{i}}\left(U_{j}\left(s_{i}^{\prime}, \mathbf{s}_{-i}\right)-U_{j}(\mathbf{s})\right)+\sum_{j \notin \mathcal{N}_{i}}\left(U_{j}\left(s_{i}^{\prime}, \mathbf{s}_{-i}\right)-U_{j}(\mathbf{s})\right) \\
& =\sum_{j \in \mathcal{N}_{i}}\left(U_{j}\left(s_{i}^{\prime}, \mathbf{s}_{-i}\right)-U_{j}(\mathbf{s})\right) \\
& =V_{i}\left(s_{i}^{\prime}, \mathbf{s}_{-i}\right)-V_{i}(\mathbf{s}),
\end{aligned}
$$

where $(*)$ follows from the fact that, for all $j \notin \mathcal{N}_{i}, i \notin \mathcal{N}_{j}$ and thus $U_{j}(\mathbf{s})$ is independent of $i$ 's strategy. So, the function $U(\cdot)$ is a potential function for the game $\left\{\mathcal{N},\left(\mathcal{S}_{i}, i \in \mathcal{N}\right),\left(V_{i}, i \in\right.\right.$ $\mathcal{N})$ ). The game is thus a potential game; it is also finite which implies that the FIP property holds.

Motivated by the FIP property of the game, we propose the following asynchronous best response update algorithm (recall the discussion in Section II-A). We assume a slotted network operation, with $t=0,1,2, \ldots$ being the slot boundaries (i.e., decision epochs). Let $\mathbf{s}(t)$ be the resource allocation profile at time $t$. At time $t+1$, resource allocation (BS association, channel allocation and downlink transmit power assignment) for each user $i$ is updated with probability $\epsilon_{i} \in(0,1)$. The new allocation is chosen as follows:

$$
s_{i}(t+1) \in \underset{s_{i} \in \mathcal{S}_{i}}{\operatorname{argmax}} V_{i}\left(s_{i}, \mathbf{s}_{-i}(t)\right) .
$$

There is thus a strictly positive probability that any subset of users will undergo resource allocation updates simultaneously. This update process converges to a NE with probability 1. Also, this process does not require any coordination among BSs to ensure one by one updates.

The FIP property ensures that the above updates converge to a NE in a finite number of steps. However, recall that the resulting equilibrium may not be a global optimizer of the aggregate network utility $U(\cdot)$. To address this, we propose a learning algorithm called spatial adaptive play (also referred to as Gibbs sampling) next.

\section{Spatial Adaptive Play}

We again consider a slotted network operation. Let $\mathbf{s}(t)$ be the resource allocation profile at time $t$. At time $t+1$, resource allocation of each user $i$ is updated with probability $\epsilon_{i} \in(0,1)$. If user $i$ undergoes an update, its new allocation is chosen according to the following distribution: for all $s_{i} \in \mathcal{S}_{i}, s_{i}(t+$
1) $=s_{i}$ with probability

$$
\pi_{i}^{t+1}\left(s_{i}, \mathbf{s}_{-i}(t)\right)=\frac{e^{\frac{V_{i}\left(s_{i}, \mathbf{s}_{-i}(t)\right)}{T}}}{\sum_{s_{i}^{\prime} \in \mathcal{S}_{i}} e^{\frac{V_{i}\left(s_{i}^{\prime}, \mathbf{s}_{-i}(t)\right)}{T}}} .
$$

Recall that $T$ here is called temperature in statistical mechanics literature, and is decreased appropriately with time (e.g., as $T(t)=1 / \log (1+t)$ ). Following the discussion in Section II-B, this update process is guaranteed to converge to a resource allocation profile that globally maximizes the aggregate network utility $U(\cdot)$.

Remark 4.2: Our problem formulation handles joint resource allocation problem of BS association, channel assignment and power control. However, it can be easily adapted to some special cases, e.g., for BS association and power control in reuse-1 cellular networks.

Remark 4.3: In the proposed joint resource allocation algorithm, a strategy could be updated one component at a time. More precisely, once a user $i$ is chosen for update, either its BS association or channel allocation or downlink power could be updated. This significantly reduces the computational requirement at each iteration. Borst et al. [24] refer to this modification as Constrained Gibbs Sampler (CGS) algorithm.

\section{INFORMATION EXCHANGE AND THE DISTRIBUTED PROTOCOL}

\section{A. Information Exchange}

Recall that the joint resource allocation profile is $\mathbf{s}(t)$ just prior to $t+1$, and at $t+1$ each user $i$ 's allocation is updated with probability $\epsilon_{i}$. Let us tag a user $i$ that is chosen for update. To implement this update $V_{j}\left(s_{i}^{\prime}, \mathbf{s}_{-i}(t)\right)$ must be computed for each $s_{i}^{\prime}=\left(b_{i}^{\prime}, c_{i}^{\prime}, p_{i}^{\prime}\right) \in \mathcal{S}_{i}$ and $j \in \mathcal{N}_{i}$ (including $i$ ), which in turn requires that $\operatorname{SINR}_{j}\left(s_{i}^{\prime}, \mathbf{s}_{-i}(t)\right)$ be computed for each $s_{i}^{\prime} \in \mathcal{S}_{i}$ and $j \in \mathcal{N}_{i}$. We assume that this computation takes place at $b_{i}(t)$, the current BS of $i$. In order to compute these quantities, $b_{i}(t)$ needs certain information from it users and also from the neighboring BSs (see Section IV-A for the notion of neighboring BSs).

We first describe the information flow to $\mathrm{BS} b_{i}(t)$ from its current users as well as from its neighbors. We also illustrate the computation of $\operatorname{SINR}_{j}\left(s_{i}^{\prime}, \mathbf{s}_{-i}(t)\right)$ for each $s_{i}^{\prime} \in \mathcal{S}_{i}$ and $j \in \mathcal{N}_{i}$ at BS $b_{i}(t)$. For clarity, we drop the time argument in the following.

1) Information sent to $b_{i}$ by each $j$ with $b_{j}=b_{i}$ :

1) The path loss (power attenuation) $l\left(j, b_{j}^{\prime}, c_{j}^{\prime}\right)$ for each BS $b_{j}^{\prime} \in \mathcal{B}_{j}$ and channel $c_{j}^{\prime} \in \mathcal{C}$ (We assume that the BSs know $\alpha\left(c, c^{\prime}\right)$ for all $\left.c, c^{\prime} \in \mathcal{C}\right)$.

2) The total received interference (including receiver noise) $I_{j}\left(s_{j}^{\prime}, \mathbf{s}_{-j}\right)+w_{j}\left(c_{j}^{\prime}\right)$ for each $b_{j}^{\prime} \in \mathcal{B}_{j}$ and $c_{j}^{\prime} \in \mathcal{C}{ }^{4}$

Clearly, $b_{i}$ can compute $\operatorname{SINR}_{i}\left(s_{i}^{\prime}, \mathbf{s}_{-i}\right)$ for any $s_{i}^{\prime} \in \mathcal{S}_{i}$. For any $j \neq i$ with $b_{j}=b_{i}$, and for any $s_{i}^{\prime} \in \mathcal{S}_{i}$,

$$
\begin{aligned}
I_{j}\left(s_{i}^{\prime}, \mathbf{s}_{-i}\right)=I_{j}(\mathbf{s}) & -\alpha\left(c_{j}, c_{i}\right) l\left(j, b_{i}, c_{i}\right) p_{i} \\
& +\alpha\left(c_{j}, c_{i}^{\prime}\right) l\left(j, b_{i}^{\prime}, c_{i}^{\prime}\right) p_{i}^{\prime} \quad\left\{b_{i}^{\prime} \in \mathcal{B}_{j}\right\} .
\end{aligned}
$$

${ }^{4}$ Observe that $I_{j}\left(s_{j}^{\prime}, \mathbf{s}_{-j}\right)$ depends on $s_{j}^{\prime}=\left(b_{j}^{\prime}, c_{j}^{\prime}, p_{j}^{\prime}\right)$ only through $b_{j}^{\prime}$ and $c_{j}^{\prime}$. 
Hence, $b_{i}$ can compute $I_{j}\left(s_{i}^{\prime}, \mathbf{s}_{-i}\right)+w_{j}\left(c_{j}\right)$ for each $j \neq i$ with $b_{j}=b_{i}$ and for each $s_{i}^{\prime} \in \mathcal{S}_{i}$. Subsequently, it can also compute $\operatorname{SINR}_{j}\left(s_{i}^{\prime}, \mathbf{s}_{-i}\right)$ for each $j \neq i$ with $b_{j}=b_{i}$ and for each $s_{i}^{\prime} \in \mathcal{S}_{i}$.

2) Information sent to $b_{i}$ by each neighboring $B S b^{\prime}$ about each $j$ with $b_{j}=b^{\prime}$ :

1) The received signal power $l\left(j, b_{j}, c_{j}\right) p_{j}$.

2) The path loss (power attenuation) $l\left(j, b_{j}^{\prime}, c_{j}^{\prime}\right)$ for each BS $b_{j}^{\prime} \in \mathcal{B}_{j}$ and channel $c_{j}^{\prime} \in \mathcal{C}$.

3) The total received interference (including receiver noise) $I_{j}(\mathbf{s})+w_{j}\left(c_{j}\right)$.

BS $b^{\prime}$ obtains this information from its users as described earlier. Now consider any user $j \in \mathcal{N}_{i}$ with $b_{j}(t) \neq b_{i}$. Then $b_{j}(t)$ must be a neighbor of $b_{i}$ (see Section IV-A). For any $j$ for which $b_{j}(t)=b^{\prime}$ is a neighbor of $b_{i}$, and for any $s_{i}^{\prime} \in \mathcal{S}_{i}$,

$$
\begin{array}{rlc}
I_{j}\left(s_{i}^{\prime}, \mathbf{s}_{-i}\right)=I_{j}(\mathbf{s}) & -\alpha\left(c_{j}, c_{i}\right) l\left(j, b_{i}, c_{i}\right) p_{i} & \left\{b_{i} \in \mathcal{B}_{j}\right\} \\
& +\alpha\left(c_{j}, c_{i}^{\prime}\right) l\left(j, b_{i}^{\prime}, c_{i}^{\prime}\right) p_{i}^{\prime} & \left\{b_{i}^{\prime} \in \mathcal{B}_{j}\right\}
\end{array}
$$

As before, $b_{i}$ can compute $I_{j}\left(s_{i}^{\prime}, \mathbf{s}_{-i}\right)+w_{j}\left(c_{j}\right)$, and subsequently $\operatorname{SINR}_{j}\left(s_{i}^{\prime}, \mathbf{s}_{-i}\right)$, for each $j$ with $b_{j}=b^{\prime}$ and for each $s_{i}^{\prime} \in \mathcal{S}_{i}$. Note that user $j$ 's $\operatorname{SINR}, \operatorname{SINR}_{j}(\cdot)$, remains unchanged if neither $b_{i} \in \mathcal{B}_{j}$ nor $b_{i}^{\prime} \in \mathcal{B}_{j}$.

Thus $b_{i}(t)$ can compute $\operatorname{SINR}_{j}\left(s_{i}^{\prime}, \mathbf{s}_{-i}(t)\right)$ for each $s_{i}^{\prime} \in \mathcal{S}_{i}$ and $j \in \mathcal{N}_{i}$.

\section{B. Distributed Protocol}

We illustrate the proposed distributed protocol through Algorithm 1 where we demonstrate its execution at BS $b$. Each user $i$ undergoes a resource update after every $d_{i}$ slots where $d_{i}$ is a geometrically distributed random variable with parameter $\epsilon_{i}$. Here we discuss the complexity and convergence of this protocol.

1) Communication and computation complexity: Observe that each user $i$ needs to report $O(|\mathcal{B}||\mathcal{C}|)$ scalers to its BS, whereas each BS needs to communication $O(|\mathcal{B}||\mathcal{C}|)$ scalers corresponding to each of its users, to each of its neighbors. Thus the total number of quantities to be exchanged in each round is of order $O(|\mathcal{N}||\mathcal{B}||\mathcal{C}|)$. In practice, the amount of communication needed will be much lesser, because the number of users associated with BS and the number of BSs with whom a user can associate will be much lesser than $|\mathcal{N}|$ and $|\mathcal{B}|$ respectively.

For each strategy update, of say user $i$, BS $b_{i}$ needs to compute precisely $\left|\mathcal{N}_{i}\right|\left|\mathcal{B}_{i}\right||\mathcal{C} \| \mathcal{P}|$ utilities corresponding to $i$ 's $\left|\mathcal{N}_{i}\right|$ neighbors. Then computing the probability law for the strategy selection incurs $\left|\mathcal{B}_{\mid}\right| \mathcal{C}|| \mathcal{P} \mid$ computations. Thus the overall order of computation per update is $O(|\mathcal{N}\|\mathcal{B}|| \mathcal{C}\| \mathcal{P}|)$.

2) Convergence time: Simulated annealing algorithms (e.g., spatial adaptive play proposed in this work) usually suffer from slow convergence. There are many speed-up techniques proposed in literature, e.g., evaluating only the difference of payoff functions rather than their absolute values and twostaged simulated annealing with a fast heuristic at higher temperatures followed by a traditional simulated annealing algorithm at lower temperatures (see [28] for a discussion). The convergence speed and the quality of final solution are

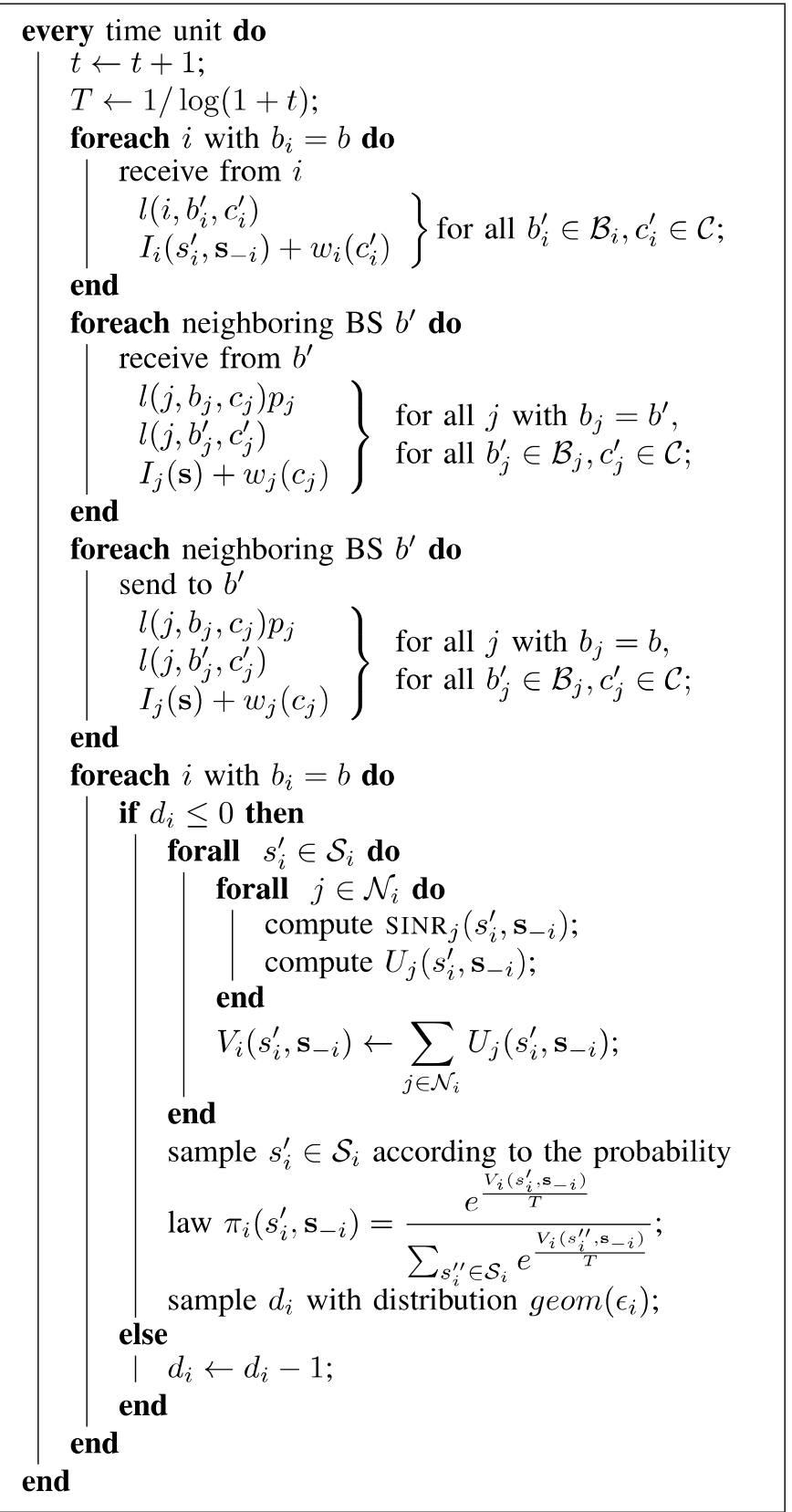

Algorithm 1: Distributed protocol running at BS $b$

also affected by the cooling function. Many heuristic methods are available in the literature to find adaptive cooling schedules which adjust the temperature's rate of decrease from information obtained during the algorithm's execution. However, we employ a traditional simulated annealing with static logarithmic cooling in our protocol.

As remarked earlier, the strategies could be updated one component at a time (in stead of updating the entire triplet). This greatly reduces the amount of communication and computation needed at each iteration, but could adversely affect the convergence time. Exploring this complexity vs. convergence time tradeoff is a subject of our future research. 

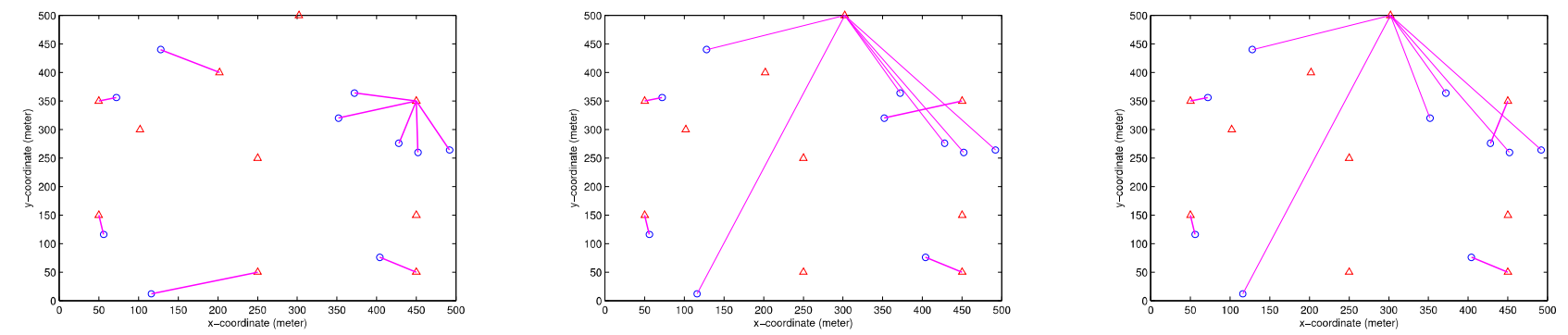

(a) Default resource allocation as per 3GPP stan- (b) Best response based algorithm for SRM objec- (c) Spatial adaptive play based algorithm for SRM dard

tive

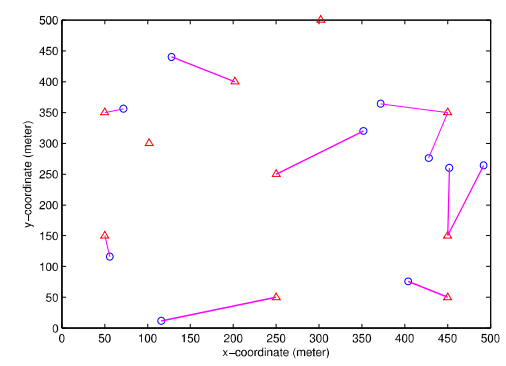

(d) Best response based algorithm for PF objective

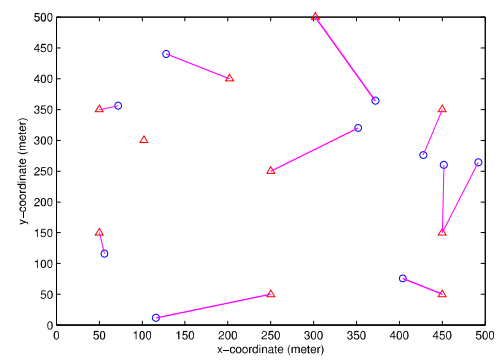

e) Spatial adaptive play based algorithm for PF objective

Fig. 1. Demonstration of the proposed algorithms over a sample pico-cell network. The users and the BSs are shown using circles ('o') and triangles $(' \Delta ')$ respectively. User-BS associations are shown using lines connecting them; a line's thickness is proportional to the downlink transmit power used by the associated BS.

We also point out that greedy version of the proposed algorithm (i.e., the one based on best response strategy) exhibits much faster convergence, and yields performance close to the optimum (see the numerical results in Section VI).

\section{Numerical StUdies}

In the following, we present simulation results. We begin with a description of network scenario and simulation setup. Then we demonstrate the performance of the proposed resource allocation algorithms.

Following 3GPP-LTE standard and default operations, currently BSs are configured to transmit with a nominal fixed power such that the pilot signal could be received by mobile users [29]. The downlink transmit power is usually the maximum allowable power for a better user reception and coverage. The BSs periodically broadcast pilot signals such that users' transceivers can perform channel measurements and appropriate tuning from time to time. As for BS association, the current practice attaches a user to a BS from which the received signal strength is strongest. This may result in a BS being overloaded if this BS is the closest BS to too many users, which may be sub-optimal. Finally, we assume in our simulation that the BSs operate in same band, and do not exercise channel selection (as in the case of reuse-1 cellular networks). However, recall that the proposed protocols are capable of jointly handling downlink power control, BS assignment and subcarrier allocation.

In our simulation, we consider a dense urban cellular network where some pico-cells are randomly located in a geographical area of $500 \mathrm{~m} \times 500 \mathrm{~m}$. Users are uniformly distributed in this area. We adopt the 3GPP-3GPP2 pico-cell spatial channel model [30]. The distance dependent path loss is given by

$$
P L^{(\mathrm{dB})}(d)=-\left(30.6+36.7 \log _{10}(d)+X_{\sigma}^{(\mathrm{dB})}\right),
$$

where $d$ is the transmitter-receiver distance in meters and $X_{\sigma}$ refers to log-normal shadowing with zero mean and standard deviation $10 \mathrm{~dB}$. We consider system bandwidth $10 \mathrm{MHz}$, noise density $-174 \mathrm{dBm} / \mathrm{Hz}$ and noise figure $9 \mathrm{~dB}$. The noise powers at all users' receivers are assumed to be $-95 \mathrm{dBm}$. The maximum transmit power of BS is $1 \mathrm{~W}$. We assume that BSs power levels are discretized in steps of $0.1 \mathrm{~W}$ and also that a BS's least transmission power to an associated user is $0.1 \mathrm{~W} .^{5}$ Recall that the BSs are using the same channel.

We implement both the proposed algorithms, the best response based algorithm (see Section IV-B) and the Gibbs sampling based algorithm, i.e., spatial adaptive play (Section IV-C). We also consider two cases of utility functions: in the first case the objective is to maximize the sum of rates of all the users whereas in the second case the goal is to optimize the sum of $\log ($ rate) of all the users (see Section III-B for a discussion on the utility functions). ${ }^{6}$ We refer to these cases as sum rate maximization (SRM) and proportional fairness (PF) in the following.

\footnotetext{
${ }^{5}$ Thus we exclude the possibility of BSs using zero power, though our framework does not require this condition and allows all non-negative powers.

${ }^{6}$ Again note that the proposed protocols can work in a more general setup where different users may have different and arbitrary utility functions
} 


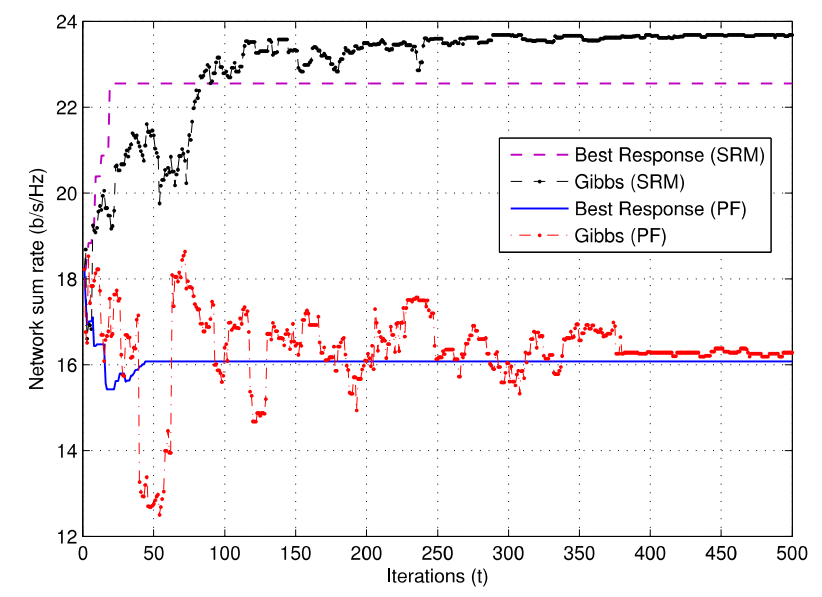

Fig. 2. Convergence of the best response based and Gibbsian algorithms and their aggregate throughput performance

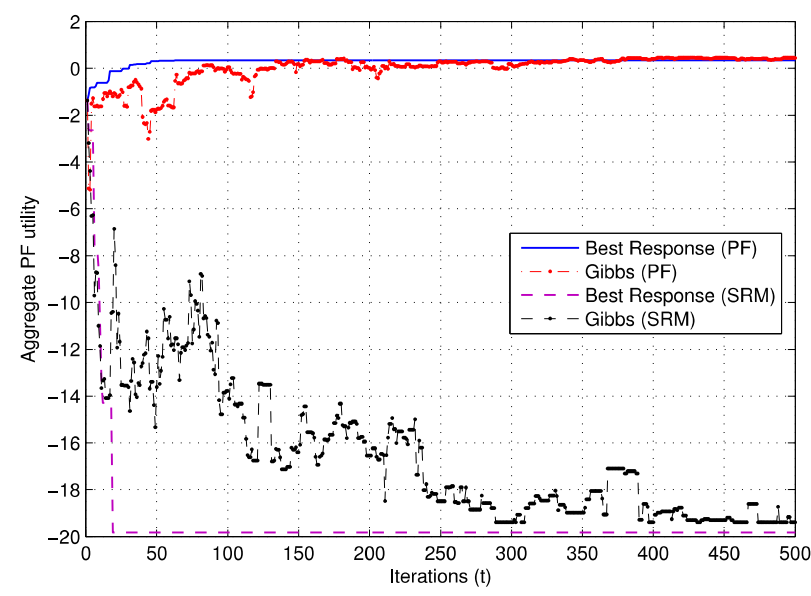

Fig. 3. Proportional fairness utility yielded by best response based and Gibbsian algorithms

We now demonstrate the performance of the proposed algorithms over a pico-cell network with $10 \mathrm{BSs}$ and 10 users (see Figure 1). The BSs and the users are deployed as described earlier. To better illustrate the effectiveness of the proposed algorithms, we only consider path loss attenuations in this simulation, i.e., we ignore log-normal shadowing components. A line connecting a user and a BS indicates a user-BS association and its thickness represents the downlink transmit power used by the corresponding BS. Subfigure (a) shows the default resource allocation (BS association and downlink transmit power) prescribed by 3GPP standard. Then we consider SRM objective; Subfigures (b) and (c) demonstrate the resource allocations led to by the best response based and Gibbsian algorithms respectively. Finally, Subfigures (d) and (e) show the BS associations and transmit powers led to by the best response based and Gibbsian algorithms algorithms respectively, for the case of $\mathrm{PF}$ objective.

Figure 2 illustrates the convergence of the best response

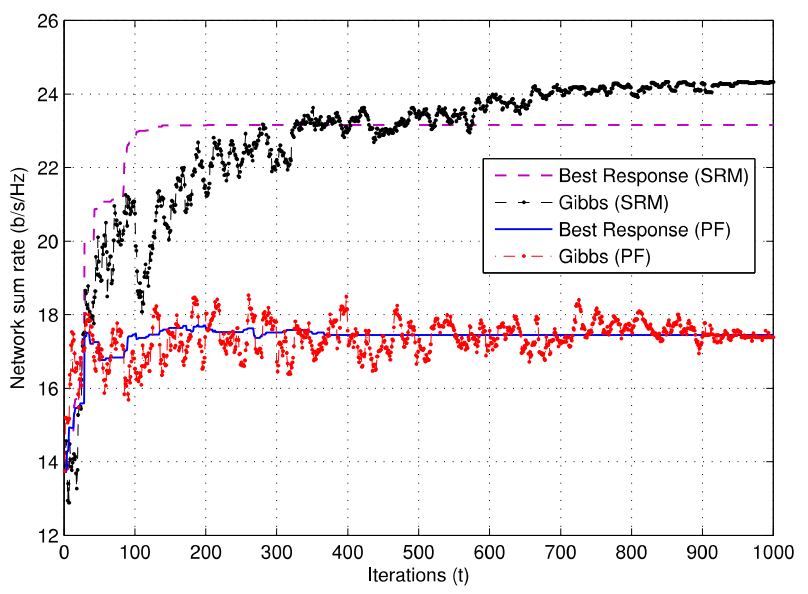

Fig. 4. Convergence of the best response based and Gibbsian algorithms and their aggregate throughput performance

based and Gibbs algorithms run over the network topology of Figure 1. In each case (i.e., for each algorithm-utility function combination), the initial resource allocation profile is that prescribed by 3GPP standard. Thus the default network performance (as per the current standard) is that corresponding to $t=0$ in the figure. We observe that, for either objective, the optimal distributed algorithm (spatial adaptive play) converges after 400 iterations, while best response based deterministic algorithm takes less than 50 iterations. With sum rate optimization objective in place, the aggregate throughput has been increased substantially by best response based and Gibbsian algorithms by $24 \%$ and $30 \%$, respectively.

Figure 3 shows the proportional fairness utility yielded by best response based and Gibbsian algorithms. These algorithms have improved the network utility from -1.37 to 0.43 and 0.48 , respectively. Quite expectedly, the resource allocations in the case of sum rate maximization are highly unfair, and thus result in poor network utilities.

We next explore the effect of network size on the convergence speed of the proposed algorithms. Towards this. we double the network density; we now have 20 BSs and 20 users placed over the same geographical area of $500 \mathrm{~m} \times 500 \mathrm{~m}$. We observe that the number of iterations required by the optimal Gibbsian algorithm is also almost doubled (see Figure 4); 800-850 iterations are required now as compared to the 400 iterations required earlier (in Figure 2). This indicates that the convergence time scales almost linearly with the network size. We also observe that, in the case of sum rate optimization, best response based and Gibbsian algorithms increase the network throughput by $68 \%$ and $77 \%$, respectively. So, the proposed algorithms yield higher performance gains in larger networks.

We make an interesting observation from these plots. For either objective function, performance of the best response based deterministic algorithm is quite close to that of the optimal Gibbsian algorithm (spatial adaptive play), and the performance gap further reduces as the network size increases. Also, the deterministic algorithm converges very quickly. This 
makes it quite useful in practice, where network topologies and channel conditions keep changing, and any good algorithm needs to adapt at a fast time scale. Deriving bounds on suboptimality of best response based algorithm (i.e., bounds on the performance gap with respect to the optimal algorithm) is challenging problem, and is a subject of our future research.

\section{CONCLUSION}

In this paper, we developed distributed algorithms for selfoptimization of cellular networks using potential games and spatial adaptive play (SAP). We would like to reemphasize that the connection between potential games and Gibbs sampling is quite generic, and it can be exploited to address further extensions of our problem and in other contexts as well. We next aim to use this framework to study enhanced inter-cell interference coordination (eICIC) schemes proposed for mboxLTEA heterogeneous networks [31]. In particular, we will focus on optimization of power bias used in cell selection (also termed as cell range extension) and on design of almost blank subframes (ABS) to combat the heavy interference imposed by macro cells on small cell edge users. It would also be interesting to extend this framework to develop joint spectrum and energy efficient protocols for $4 \mathrm{G}$ and beyond systems.

We assumed constant channel gains in our analysis and we let the Gibbs sampling based algorithm run long enough to ensure convergence. In practice, channel gains vary with time. If we run the simulated annealing for long duration, we obtain optimal resource allocation with very probability, but we also risk using this allocation in a network where channel gains have significantly changed. On the other hand, if we terminate the simulated annealing prematurely, we may endup with a sub-optimal resource allocation with nonnegligible probability. Recall that performance of simulated annealing is also affected by the cooling function. Joint study of wireless channel dynamics, cooling procedure and the execution time of simulated annealing is a challenging research avenue.

\section{ACKNOWLEDGMENT}

This work was carried out at LINCS (www.lincs.fr) and under INRIA-Alcatel-Lucent Bell Labs Joint Research Center. The research leading to these results has received funding from European CELTIC-Plus under project OPERA-Net2. We would like to thank Laurent Roullet, Bruno Gaujal and Vinod Kumar for their valuable discussions and comments.

\section{REFERENCES}

[1] Next Generation Mobile Networks Group. http://www.ngmn.org

[2] 3rd Generation Partnership Project. 3GPP-LTE. http://www.3gpp.org.

[3] M. Chiang, C. W. Tan, D. P. Palomar, D. O'Neill, and D. Julian, "Power control by geometric programming," IEEE Transactions on Wireless Communications, vol. 6, no. 7, pp. 2640-2651, July 2007.

[4] C. S. Chen and G. E. Oien, "Optimal power allocation for two-cell sum rate maximization under minimum rate constraints," in Proceedings of IEEE International Symposium on Wireless Communication Systems, 2008, pp. 396-400.

[5] G. J. Foschini and Z. Miljanic, "A simple distributed autonomous power control algorithm and its convergence," IEEE Transactions on Vehicular Technology, vol. 42, no. 4, pp. 641-646, November 1993.

[6] R. D. Yates, "A framework for uplink power control in cellular radio systems," IEEE J. Select. Areas Commun., vol. 13, no. 7, pp. 1341-1347, September 1995.
[7] S. V. Hanly, "An algorithm for combined cell-site selection and power control to maximize cellular spread spectrum capacity," IEEE J. Select. Areas Commun., vol. 13, no. 7, pp. 1332-1340, September 1995.

[8] F. Rashid-Farrokhi, K. J. R. Liu, and L. Tassiulas, "Downlink power control and base station assignment," IEEE Communications Letters, vol. 1, no. 4, pp. 102-104, July 1997.

[9] F. Baccelli, N. Bambos, and N. Gast, "Distributed delay-power control algorithms for bandwidth sharing in wireless networks," IEEE Transactions on Networking, vol. 19, no. 5, pp. 1458-1471, October 2011.

[10] T. Bu, L. E. Li, and R. Ramjee, "Generalized proportional fair scheduling in third generation wireless data networks," in Proceedings of IEEE INFOCOM, Barcelona, Spain, April 2006.

[11] J. Huang, V. Subramanian, R. Agrawal, and R. Berry, "Downlink scheduling and resource allocation for ofdm systems," IEEE Transactions on Wireless Communications, vol. 8, no. 1, pp. 288-296, January 2009.

[12] X. Wu, A. Das, J. Li, and R. Laroia, "Fractional power reuse in cellular networks," in Proceedings of Forty-Fourth Annual Allerton Conference, Champaign, Illinois, USA, September 2006.

[13] A. L. Stolyar and H. Viswanathan, "Self-organizing dynamic fractional frequency reuse in ofdma systems," in Proceedings of IEEE INFOCOM, Phoenix, AZ, USA, April 2008.

[14] I.-H. Hou and C. S. Chen, "Self-organized resource allocation in lte systems with weighted proportional fairness," in Proceedings of IEEE International Conference on Communications, Ottawa, Canada, June 2012.

[15] G. Scutari, S. Barbarossa, and D. P. Palomar, "Potential games: A framework for vector power control problems with coupled constraints," in Proceedings of IEEE ICASSP, vol. 4, May 2006, pp. IV-241-IV-244.

[16] T. Heikkinen, "A potential game approach to distributed power control and scheduling," Computer Networks, vol. 50, no. 13, pp. 2295-2311, Sept. 2006.

[17] E. Altman and Z. Altman, "S-modular games and power control in wireless networks," IEEE Trans. on Automatic Control, vol. 48, no. 5, pp. 839-842, May 2003.

[18] T. Alpcan, T. Basar, R. Srikant, and E. Altman, "CDMA uplink power control as a noncooperative game," Wireless Networks, vol. 8, no. 6, pp. 659-670, Nov. 2002.

[19] C. U. Saraydar, N. B. Mandayam, and D. J. Goodman, "Efficient power control via pricing in wireless data networks," IEEE Trans. on Communications, vol. 50, no. 2, pp. 291-303, Feb. 2002.

[20] C. Singh, A. Kumar, and R. Sundaresan, "Uplink power control and base station association in multichannel cellular networks," in Proceedings of GameNets, Istanbul, Turkey, May 2009, pp. 43-51.

[21] L. Jiang, S. Parekh, and J. Walrand, "Base station association game in multi-cell wireless networks," in IEEE Wireless Communication and Networking Conference, March 2008, pp. 1616-1621.

[22] B. Kauffmann, F. Baccelli, A. C. V. Mhatre, K. Papagiannaki, and C. Diot, "Measurement-based self organization of interfering 802.11 wireless access networks," in Proceedings of IEEE INFOCOM, Anchorage, AK, USA, May 2007.

[23] C. S. Chen and F. Baccelli, "Self-optimization in mobile cellular networks: Power control and user association," in Proceedings of IEEE International Conference on Communications, Cape Town, South Africa, May 2010.

[24] S. Borst, M. Markakisy, and I. Saniee, "Distributed power allocation and user assignment in ofdma cellular networks," in Proceedings of Forty-Ninth Annual Allerton Conference, Champaign, Illinois, USA, September 2011.

[25] D. Monderer and L. S. Shapley, "Potential games," Games and Economic Behavior, vol. 14, no. 1, pp. 124-143, May 1996.

[26] J. O. Neel, "Analysis and design of cognitive radio networks and distributed radio resource management algorithms," Ph.D. dissertation, Virginia Polytechnic Institute and State University, Sept. 2006.

[27] H. P. Young, Individual Strategy and Social Structure: An Evolutionary Theory of Institutions. Princeton University Press, 1998.

[28] L. Ingber, "Simulated annealing: Practice versus theory," Mathematical and Computer Modelling, vol. 18, no. 11, pp. 29-57, December 1993.

[29] 3GPP TS 36.331, "Evolved universal terrestrial radio access radio resource control: Protocol specification," Tech. Spec. v10.4.0, 2011.

[30] 3GPP TS 36.839, "Mobility enhancements in heterogeneous networks," Tech. Spec. v11.1.0, 2012.

[31] S. Deb, P. Monogioudis, J. Miernik, and J. Seymour, "Algorithms for enhanced inter cell interference coordination (eICIC) in LTE HetNets," IEEE Transactions on Networking, vol. accepted, March 2013. 